\title{
The acquisition of temporal categorical perception by Japanese second language learners
}

\author{
Naoko Kinoshita \\ Integrated Education Center, Meikai University, Japan \\ https://doi.org/10.36505/ExLing-2008/02/0031/000090
}

\begin{abstract}
This study reports research into the development of perception of special mora categories by Korean learners of Japanese as a second language. First, a test which measured boundary width and the boundary point of distinction between singletons and special mora was used to establish participants' categorical perception across four levels of proficiency. Then a two year longitudinal study followed the development of 14 learners, during which time the same test was administered three times. The results demonstrated: 1) that the special mora perceptual categories of second language learners developed in some areas, but not in others and 2) that there was a categorization of the special mora over time.
\end{abstract}

Key words: categorical perception, rhythm, language acquisition, mora timing

\section{Introduction}

Japanese is a mora-timed language in which each mora receives roughly the same articulation time. There are three special mora (long vowels, geminate consonants, and nasal consonants) perceived to receive twice as much articulation time. For example, while obasan means 'aunt' in Japanese, oba:san means 'grandmother'. This distinction is difficult for learners of Japanese to acquire. As yet, there has been little research which examines the acquisition of the mora-timed rhythm. (However, see Kinoshita 2007, Nishogori et al. 2002, Toda 1998, and Uchida 1998)

This study attempts to determine the process of acquisition of categorical perception of special mora by Korean learners of Japanese. It approaches the problem using both cross-sectional and longitudinal methodologies.

\section{Research Method \\ Participants}

The participants were 21 Korean university students who were studying Japanese at a university in Busan. Based on the Oral Proficiency Interview (OPI) held at the beginning of the study, these included 6 advanced learners, 9 intermediate and 6 beginning learners. Of these 21, 14 learners participated in the longitudinal study. In addition to the Korean learners of Japanese, 7 Japanese native speakers of the Tokyo dialect (JS) were involved in the study. All participants were compensated for their time.

ExLing 2008: Proceedings of 2nd Tutorial and Research Workshop on Experimental Linguistics, 25-27 August 2008, Athens, Greece 


\section{Instruments and Procedure}

In order to measure the participants' perception of Japanese mora rhythm, a perceptual experiment was designed. Eight minimal pairs were recorded by a female native speaker of the Tokyo dialect in meaningful carrier sentences. Two sentences contrasted the long/short vowel distinction (R), two contrasted the nasal /N/ distinction $(\mathrm{N})$ and four contrasted the geminate/singleton consonant distinction (Q). The selection of the minimal pairs was also controlled for accent (HL and LH) and, in the case of the geminate/singleton, consonant (fricative and plosive) (see Toda 1998).

Fifteen samples of each minimal pair were then generated from the natural recording using acoustic analysis software (OnseiKoubo NTT Advanced Technology). The vowels, nasals and consonants were lengthened at increments of $10 \%$ from $-20 \%$ to $+120 \%$ with $0 \%$ being the length of the short sound, and $100 \%$ being the length of the long sound.

These samples were presented to the participants on an audio CD, twice, and in random order. They were required to indicate if the stimulus word in each sentence was long or short by circling the word. This resulted in 240 judgements per participant. The entire procedure took about 11 minutes per CD. For the 14 longitudinal study participants, the data was collected twice more at yearly intervals between collections.

\section{Analysis}

The distinction between the temporal categories was operationalized by boundary point (BP) and boundary width (BW). BP is the point of time at which the perception of whether the sound is long or short changes. It is recorded at the point where the participants are $50 \%$ certain that the sound is long. BW is the temporal width. This is the time taken between participants' $20 \%$ certainty that the sound is long to their $80 \%$ certainty of it. BP and BW were measured for each participant and each sentence.

In order to respond to the research questions, four repeated measures ANOVAs were undertaken. The first two compared level (NS, beginner, intermediate and advanced) with rhythm type (R, N and Q) for both BP and BW. Similarly, the second two compared the time (year 1, year 2, and year 3 ) with the rhythm types (R, N, and Q). Significance was set at 5\%.

\section{Results}

First, there were no significant differences between the four proficiency levels for R. However, the Korean groups did have boundary points significantly displaced from those of Japanese native speakers for $\mathrm{N}$ and Q. There were no differences between Korean learners' proficiency levels. However, there was a proficiency level difference for Boundary Width. The 
beginners perceived larger BWs for all three rhythm types than the intermediate and advanced groups. There were no observed differences between the other learner groups and JS. These results are represented visually in figures 1 and 2 .
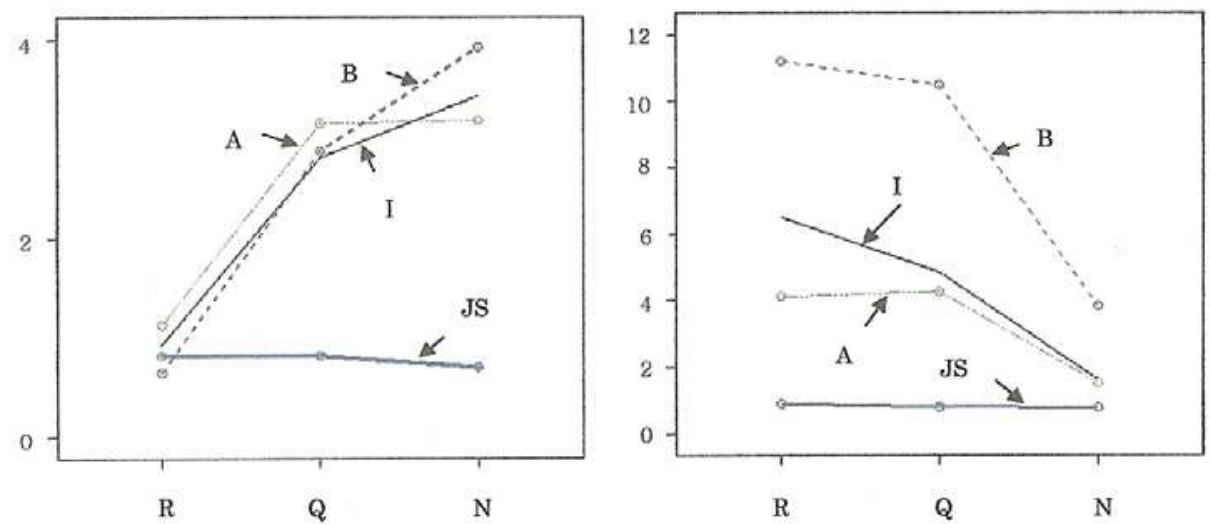

Figures 1 and 2. The BP and BW perception of R, Q and N (B = beginning learners, $\mathrm{I}=$ intermediate learners, $\mathrm{A}=$ advanced learners, $\mathrm{JS}=$ Japanese native speakers).
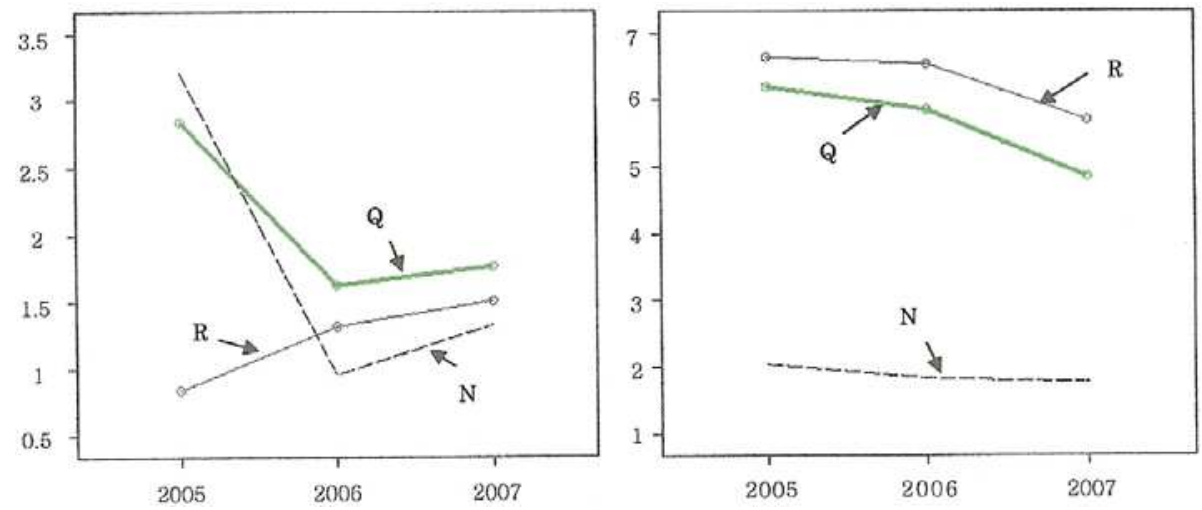

Figures 3 and 4. The change in $\mathrm{BP}$ and $\mathrm{BW}$ perception of R, Q and $\mathrm{N}$ over time.

The BP longitudinal data demonstrated a trend toward a general rhythmic perceptual category. Initially, in 2005 , R was very close to the JS score. However, by 2006 it had significantly increased from its 2005 value, away from JS perception. In contrast, the BPs of Q and N become significantly closer to that of the JS from 2005 and 2006. In both 2006 and 2007 there was no significant difference between the R, Q or N. There was no change for BW over the three times it was measured in the cross sectional study and the 
significantly narrower BW demonstrated in the longitudinal data was maintained (see figures 3 and 4).

\section{Discussion and Conclusion}

The analyses of the results have provided us with several points of discussion. First, there does appear to be some developmental change in the perception of rhythm. This is evident in both the BW data in the crosssectional results and the BP data in the longitudinal study. These results mirror those of Kinoshita (2007) and Nishigori et al. (2002) However, even over the two year duration of this study, there were areas of perception which did not change, indicating that these aspects of rhythm may need to be taught to learners.

Perhaps the most interesting result was that the Korean learners' boundary point timing showed they had 'categorized' (see Ryalls 2003, p. 44) the special mora. Initially the BPs were different for the three special mora, however, in the second and third year of the study, they moved closer together, with R even moving away from the JS norm.

\section{Acknowledgements}

This work was funded in part by the Grant-in-Aid for Scientific Research (C), "The acquisition of Japanese special mora by second language learners", no. 19520463 from JSPS.

\section{References}

Kinoshita, N. 2007. The Acquisition Processes of the Perception of Japanese Long and Short Vowels: The Case of Korean Learners of Japanese as a Second Language, The Korean Journal of Japanology. 72, 1-12.

Nishigori, J., Hwan, Y., Park, Y. 2002. Kankokujin gakusyusya no Nihongo sokuon no chikaku ni kansuru kenkyu: gakusyu reberubetsu tokusei to bogo niyoru setsumei no kouka, Nihongo Kenkyu22, 103-118.

Ryalls, J. 1996. A Basic Introduction to Speech Perception, Tokyo, Kaibundo.

Toda, T. 1998. Perceptual categorization of the durational contrasts by Japanese learners. Studies in Language and Literature 33, University of Tsukuba, 65-82.

Uchida, T. 1998. Categorical Perception of Relatively Steady-Static Speech Duration in Japanese Moraic Phonemes, Journal of Phonetic Society of Japan 2-3, 71-86. 